\title{
The Effect of Partitioning on the Clustering Index of Randomly-Oriented Fiber Composites: A Parametric Study
}

\author{
Zia Javanbakht ${ }^{\mathrm{a}}$, Wayne Hall ${ }^{\mathrm{b}}$, Andreas Öchsner ${ }^{\mathrm{c}}$
}

School of Engineering, Griffith University, Gold Coast Campus, Southport 4222, Australia.

a Zia.Javanbakht@gmail.com, b w.hall@griffith.edu.au , ${ }^{\mathrm{c}}$ Andreas.Oechsner@gmail.com

Keywords: Finite element method; Reinforced composites; Thermal analysis; Fiber clustering.

\begin{abstract}
In the current study, five cases of fiber distributions are considered in a fiber-reinforced composite: one random, three partitioned (one uniform and two biased cases), and one aligned case for benchmarking. The finite element method and the principal component analysis were used to interpret the results of orientation tensors and detect any possible clusterings of a representative volume element (RVE). The obtained effective conductivity values were extensively controlled by the fiber volume fraction. At the same time, the uniformity of the random distributions could be recognized. Cross-partition resistance was also detected for the partitioned cases which contributed to a reduced heat transfer capability. Finally, the clustering indexes did not show a direct correlation with the conductivity results, and thus a case-by-case investigation is recommended to consider the anisotropic aspects of a microstructure.
\end{abstract}

\section{Introduction}

A combination of two or more materials - distinct in properties and boundaries - is referred to as a composite material. The material with the greatest volume is the matrix while other constituents are generally called inclusions. The properties of the final material depend on the properties of all its constituents; geometry, distribution, orientation, and concentration of the reinforcements; and the quality of the matrix-inclusion interface [1]. Inclusions are used in the form of fibers, laminates, particles, or a combination of them to produce various classes of composites [2] among which randomly-oriented fibers are of interest in the current study.

Various analytical, numerical and statistical approaches are proposed in the literature to foresee the properties of a composite. In most of such procedures a randomly-oriented - yet uniform - distribution of fibers is assumed, see for instance $[3,4,5]$. However, under some practical manufacturing conditions, e.g., poor mixing in injection molding, or high volume fraction of fibers, uniformity of dispersion is lost [6]. Namely, fiber-fiber interactions within the composite may affect the dispersion state, and create non-uniform concentration regions of fibers. In such a domain with non-homogeneous fiber distribution, fibers in the high concentrations regions co-orient themselves, and thus a preferred orientation is formed - called fiber clustering, albeit particle clustering is also possible $[7,8,9]$.

In terms of direction, an orientation cluster is formed by the fibers of a region which share the same orientation state [10]. Kanatani [11] introduced three frame indifferent forms for capturing the statistical directional data and tested their application on inter-particular contact distribution of a 2D granular material. Tensorial representation of fiber orientation was introduced in $[12,13]$ to ultimately quantify fiber clustering.

Orientation tensors were measured and calculated for injection-molded tensile samples which indicated a flow-direction alignment close to the mold wall while a more random dispersion exists in the middle of the specimens [14]. Such measurements are crucial for characterizing the physical properties of the specimen based on its internal structure. For instance, elastic properties of short-fibers can be extracted more accurately from homogenization of the micro-computer tomography images by observing fiber orientations [15]. Additionally, similarities of fiber alignment phenomenon between micro- and nano-scales promise an exchangeable knowledge basis which further emphasizes on the importance of the topic $[16,17]$. 
Herein, the mixing-analogy method is used to indicate the orientation clustering, first introduced in [18], by numerically examining the incorporated tensorial representation. Four cases of moderate fiber volume fractions for randomly-oriented composites were considered by means of a planar prototype as well as an aligned model for benchmarking. A high contrast property is assumed for the composite constituents to take into account the sensitivity of such mediums to fiber clustering [6]. A list of used symbols is also provided in Table 1.

Table 1: List of symbols

\begin{tabular}{cl}
$\gamma$ & mesh density \\
$\theta_{\mathrm{P}}$ & rotation of the principal direction transformation \\
$\phi_{\mathrm{f}}$ & volume fraction of the fiber \\
$\lambda_{i}$ & $i$-th eigenvalue \\
$\psi$ & probability distribution function \\
$\Delta T$ & prescribed temperature gradient \\
$\Delta z$ & length over which temperature gradient is formed, i.e., the distance between \\
$a$ & the boundary edges \\
$k_{\mathrm{eff}}$ & element edge length \\
$k_{\mathrm{f}}$ & effective thermal conductivity of the composite \\
$k_{\mathrm{m}}$ & thermal conductivity of the fiber \\
$A_{0}$ & cross-sectional area perpendicular to direction of the flux \\
$\dot{Q}$ & total heat flux magnitude \\
$\boldsymbol{e}_{i}$ & orthonormal unit basis \\
$\boldsymbol{e}_{i}^{\mathrm{p}}$ & orthonormal unit basis of the principal direction \\
$\boldsymbol{p}$ & fiber direction vector \\
$\boldsymbol{A}$ & orientation second-order tensor \\
$\boldsymbol{A}^{*}$ & orientation second-order tensor in the spectral form \\
$\boldsymbol{Q}$ & transformation second-order tensor \\
\hline
\end{tabular}

\section{Tensorial Representation of Planar Orientation State}

The orientation of each fiber is denoted by a unit vector $(\boldsymbol{p}=\boldsymbol{p}(\theta, \phi))$ as a 3D spatial function, see Fig. 1, which can be readily specified using the summation convention:

$$
\boldsymbol{p}=p_{i} \boldsymbol{e}_{i}, \quad i=1,2,3,
$$

where $\boldsymbol{e}_{\boldsymbol{i}}$ is the $i$-th basis unit vector of the coordinate system, and $p_{i}$ is the $i$-th component of the unit vector $\boldsymbol{p}$. This presentation reduces to summation over two indexes $(i=1,2)$ for a planar coordinate system, i.e., $\boldsymbol{p}=\boldsymbol{p}(\phi)$.

The statistical distribution of fibers in the vicinity of a unit vector is specified by the probability distribution function $(\psi=\psi(\boldsymbol{p}))$ :

$$
\psi(\theta, \phi)=\delta\left(\theta-\frac{\pi}{2}\right) \psi_{\phi}(\phi),
$$

where $\delta$ is the Dirac delta function and $\psi_{\phi}$ is the planar probability distribution function. Consequently, the 2nd-order planar orientation tensor $(\boldsymbol{A})$ can be defined as [12]:

$$
A_{i j}=\int_{0}^{2 \pi} \psi_{\phi}(\phi) p_{i} p_{j} \mathrm{~d} \phi,
$$




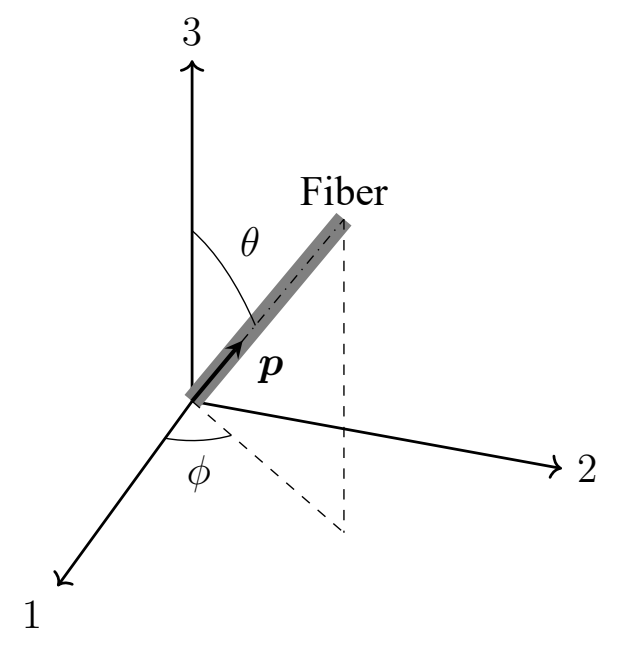

Fig. 1: Representation of the orientation of a general fiber in the 3D space using a unit vector $\boldsymbol{p}$

where $p_{i}$ and $p_{j}$ are the $i$-th and $j$-th components of the unit vector $\boldsymbol{p}$, respectively. A discretized formulation of Eq. (3) directly reveals the components of the planar orientation tensor for a specific region containing $N_{\mathrm{f}}$ number of fibers [18]:

$$
A_{i j} \approx \frac{1}{N_{\mathrm{f}}} \sum_{k=1}^{N_{\mathrm{f}}} p_{i}^{k} p_{j}^{k},
$$

where it basically provides the average orientation tensor component of the entire sample $\left(\overline{\overline{A_{i j}}}\right)$ if the total number of fibers in the sample is used. Note that since the orientation tensor is symmetric:

$$
A_{i j}=A_{j i}
$$

and to fulfill the normalization condition of $\psi_{\phi}$ :

$$
A_{i i}=1,
$$

thus there are only two independent components in the planar orientation tensor, e.g., $A_{11}$, and $A_{12}$. Namely, $A_{11}$ quantifies the proportion of alignment along the 1-axis and $A_{12}$ indicates the deviation of the coordinate axes from the principal directions of the orientation tensor.

Since the second ordered orientation tensor is symmetric, there exists an orthonormal basis $\left(\boldsymbol{e}_{i}^{\mathrm{p}}\right)$ along which the spectral form $\left(\boldsymbol{A}^{*}\right)$ of the orientation tensor $(\boldsymbol{A})$ can be acquired [19]:

$$
\boldsymbol{A}^{*}=\lambda_{i} \boldsymbol{e}_{i}^{\mathrm{p}} \otimes \boldsymbol{e}_{i}^{\mathrm{p}}
$$

where $\lambda_{i}$ are the real eigenvalues and $\boldsymbol{e}_{i}^{p}$ are the normed eigenvectors forming the basis of the orthonormal coordinate system. A unique rotation $Q$ is used to transform the orientation tensor, by an angle equal to $\theta_{\mathrm{P}}$, to the principal directions to obtain:

$$
\boldsymbol{A}^{*}=\left[\begin{array}{cc}
\lambda_{1} & 0 \\
0 & \lambda_{2}
\end{array}\right] \text {. }
$$

An ellipse can be used to illustrate the principal components. The axes of the ellipse are the principal directions and their length are proportional to the degree of the orientation along the their directions. Finally, the major axis denotes the preferred direction of the fibers. Such an ellipse can be used to graphically represent the orientation of fibers in a selected region. Note that for highly aligned fibers, the minor axis of the ellipse is diminished and only a line along the major axis will remain. In contrast, a circle indicates that no preferred directions exist $[13,12]$. 


\section{Cluster Index}

A sample of total $N_{\mathrm{f}}$ short fibers is subdivided into $N_{\mathrm{p}}$ partitions:

$$
N_{\mathrm{f}}=\sum_{l=1}^{N_{\mathrm{p}}} N_{\mathrm{f} l},
$$

where $N_{\mathrm{f} l}$ is the total number of fibers in the $l$-th partition. The orientation state of the $l$-th partition $\left(\overline{A_{i j}^{l}}\right)$ is:

$$
\overline{A_{i j}^{l}}=\sum_{k=1}^{N_{\mathrm{f} l}} A_{i j}^{k l} .
$$

and the orientation state of the whole sample is:

$$
\overline{\overline{A_{i j}}}=\sum_{k=1}^{N_{\mathrm{f}}} A_{i j}^{k} .
$$

From the mixing-analogy method, the clustering index [CI] is defined as [18]:

$$
[\mathrm{CI}]_{i j}=\frac{N_{\mathrm{p}}-1}{N_{\mathrm{f}}-1} \frac{\left[\sigma_{\mathrm{bp}}^{2}\right]_{i j}}{\left[\sigma_{\mathrm{t}}^{2}\right]_{i j}},
$$

where $\left[\sigma_{\mathrm{bp}}^{2}\right]_{i j}$ is the variance between the partitions, and $\left[\sigma_{\mathrm{t}}^{2}\right]_{i j}$ is the total variance. Finally, the type-1 clustering index can be expressed in terms of the orientation state as follows:

$$
[\mathrm{CI}]_{i j}=1-\frac{\sum_{l=1}^{N_{\mathrm{p}}} \sum_{k=1}^{N_{\mathrm{f} l}}\left(A_{i j}^{k l}-\overline{A_{i j}^{l}}\right)^{2}}{\sum_{l=1}^{N_{\mathrm{p}}} \sum_{k=1}^{N_{\mathrm{f} l}}\left(A_{i j}^{k l}-\overline{\overline{A_{i j}^{l}}}\right)^{2}},
$$

where the nominator indicates the sum of the variances within each partition and the denominator is the variance of the whole RVE.

\section{Methodology}

In the current study, the numerical solutions were obtained by means of the finite element method [20, 21, 22]. The MSC.Marc (version 2017.0) commercial package along with its Python scripting capability was used to automatize the mesh generation, job submission, and post-processing stages of the study. Moreover, object oriented programming is used to encapsulate the code for the RVE and fiber class of objects [23].

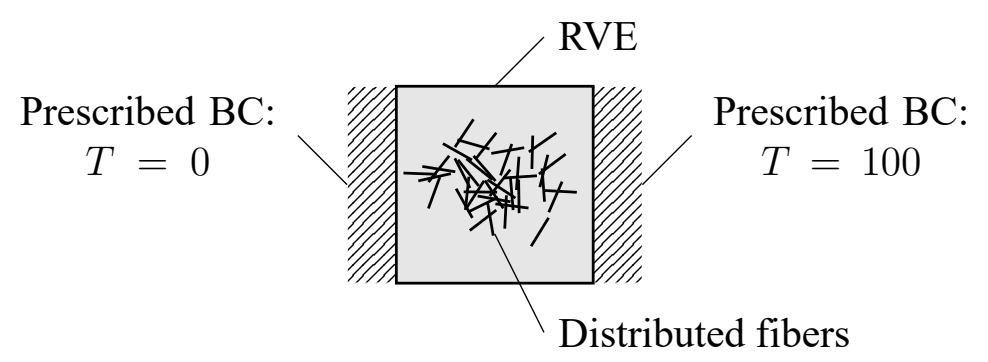

Fig. 2: Schematic illustration of the RVE prototype 
The periodicity condition was established for the $20 \times 20$ planar RVE which embedded short fibers with a length equal to 5 . Note that dimensionless values were used throughout the investigation. An aspect ratio of 40 was acquired for the fibers, i.e., a fiber length of 5 and a diameter of 0.125 , to stay within the range of 20-60 aspect ratios of the short fibers [24]. Although the analysis was carried out in the 2D space, it was assumed that the RVE has a uniform thickness equal to the diameter of the fibers. A high material contrast of 600 was assumed for the fiber to matrix thermal conductivity ratio; unit thermal conductivity was assigned to the matrix.

The boundary conditions can highly affect the results of a finite element analysis [25]. More specifically, the best results for computational analysis of a periodic composite reinforced RVE undergoing heat conduction is acquired using prescribed temperature boundary conditions [26]. Therefore, a temperature difference of 100 is applied to the right-hand boundary nodes which results in a reaction heat flux on the same edge, see Fig. 2. Fourier's law is used to calculate the effective thermal conductivity of the RVE [27]:

$$
k_{\mathrm{eff}}=\frac{\dot{Q}}{A_{0}} \cdot \frac{\Delta z}{\Delta T},
$$

where $k_{\text {eff }}$ is the effective thermal conductivity of the RVE, $\dot{Q}$ is the sum of the generated reaction fluxes on the right-hand boundary, $A_{0}$ is the assumed cross-sectional area perpendicular to direction of the flux, $\Delta z$ is the distance between the edges of the sample, and $\Delta T$ is the prescribed temperature gradient.

A planar thermal analysis was carried out for each case using a uniform mesh for the matrix consisting of type 39 four-node, isoparametric, arbitrary quadrilateral finite elements with embedded straight 2node link/truss element as fibers. The mesh sensitivity analyses were carried out for a range of mesh densities, from 1 to 35, where the uniform mesh density $\gamma$ was defined for a mesh consisting $a$ by $a$ square elements [28]:

$$
\gamma=\frac{1}{a}
$$

The prototype RVE was used to distribute the fibers based on five possible cases (Fig. 3):

1. uniform randomly-oriented without partitioning,

2. uniform randomly-oriented with partitions,

3. non-uniform randomly-oriented with partitions (downward bias),

4. non-uniform randomly-oriented with partitions (leftward bias), and

5. $30^{\circ}$ aligned fibers with respect to the horizontal axis.

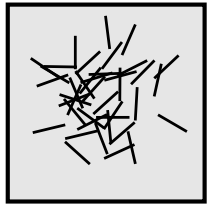

Case 1

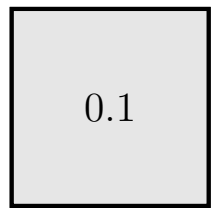

$\phi_{\mathrm{f}}$

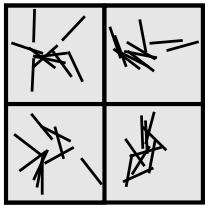

Case 2

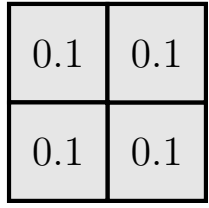

$\phi_{\mathrm{f}}$

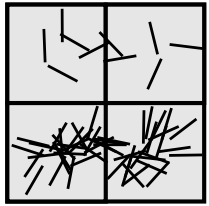

Case 3

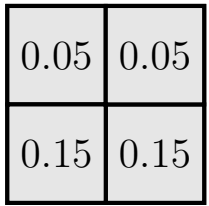

$\phi_{\mathrm{f}}$

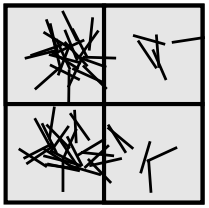

Case 4

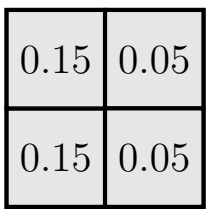

$\phi_{\mathrm{f}}$

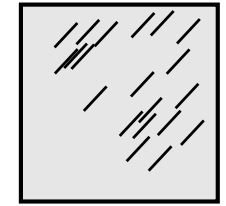

Case 5

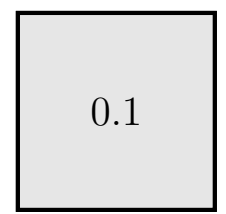

$\phi_{\mathrm{f}}$

Fig. 3: Five cases of randomly-oriented fiber distributions and one aligned case with their corresponding fiber volume fractions 


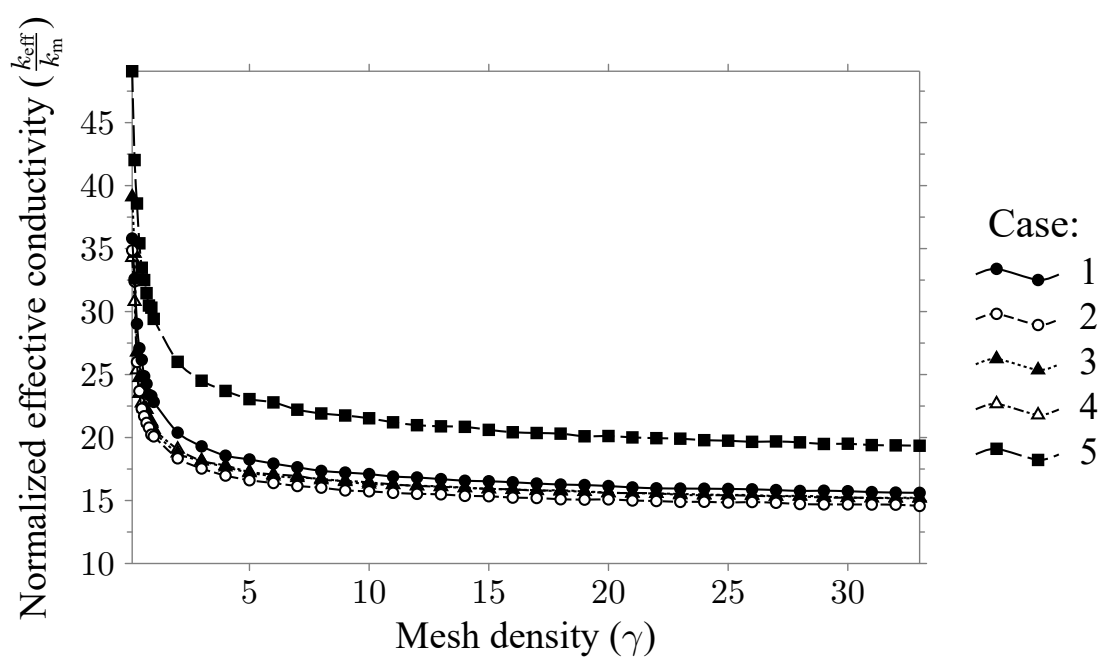

Fig. 4: Sensitivity analysis of effective thermal conductivity versus mesh density in a randomly oriented fiber distribution

The partitioning was done by dividing each edge by half resulting in four partitions in total, i.e., four $5 \times 5$ partitions. The average fiber volume fraction is the same in each case and the periodicity condition is applied to all the edges, i.e., no penetration across the inner edges in the partitioned cases is allowed. The simulations were repeated ten times for each case while the incorporated algorithm updated the fiber distribution in each job re-submission. The algorithm chooses two random coordinates for nodes, corrects the length of the fiber element, and ensures that the periodicity condition is fulfilled. This is done by cutting and transferring the excess parts of fiber elements to the appropriate edges.

\section{Results and Discussion}

The sensitivity analysis did not mark any of the five cases as critical regarding the mesh size, and thus a mesh density of 20 was chosen to conduct simulations, see Fig. 4. The temperature gradient is applied to each set and the reaction heat flux was used to calculate the dimensionless effective conductivity ratio $\left(\frac{k_{\text {eff }}}{k_{\mathrm{m}}}\right)$, see Table 2 . Case 5 is the $30^{\circ}$ aligned fibers with respect to the horizontal axis which has the highest effective conductivity. Regardless of the used meshing and partitioning method, all other cases demonstrate values in the same range denoting that the fiber volume fraction is more decisive than the type of random fiber distribution method in this case. On the other hand, case 4 results in the lowest conductivity value which is due to the fact that its leftmost partitions act as a barrier against the conduction because of their low fiber volume fractions.

All the partitioned cases demonstrate a high standard deviation which might be due to the internal edge separation of the partitions. Since the periodicity condition is also applied within each partition, no cross-partition fibers could have exist. For the same reason, no direct bridges may form between the

Table 2: Summary of the results for the parametric study

\begin{tabular}{ccccccccc}
\hline Case & $\frac{k_{\text {eff }}}{k_{\mathrm{m}}}$ & {$[\mathrm{CI}]_{11}$} & {$[\mathrm{CI}]_{12}$} & $\overline{\overline{A_{11}}}$ & $\overline{\overline{A_{12}}}$ & $\theta_{\mathrm{P}}$ & $\lambda_{\min }$ & $\lambda_{\max }$ \\
\hline 1 & 16.55845844 & 0.02350 & 0.02353 & 0.49930 & 0.02095 & 31.693540 & 0.455751 & 0.544249 \\
2 & 16.52188182 & 0.02723 & 0.02730 & 0.49875 & -0.00510 & -2.894007 & 0.446897 & 0.553103 \\
3 & 16.67016119 & 0.02772 & 0.02762 & 0.50600 & 0.00052 & 1.823605 & 0.460312 & 0.539688 \\
4 & 15.61689322 & 0.02754 & 0.02746 & 0.50932 & 0.02478 & 36.445691 & 0.446099 & 0.553901 \\
5 & 19.97653051 & 0.01437 & 0.00828 & 0.74992 & 0.43305 & 59.993894 & 0.000003 & 0.999997 \\
\hline
\end{tabular}


leftmost edge to the rightmost one in the partitioned cases which results in more dispersed conductivity values, see Fig. 5.

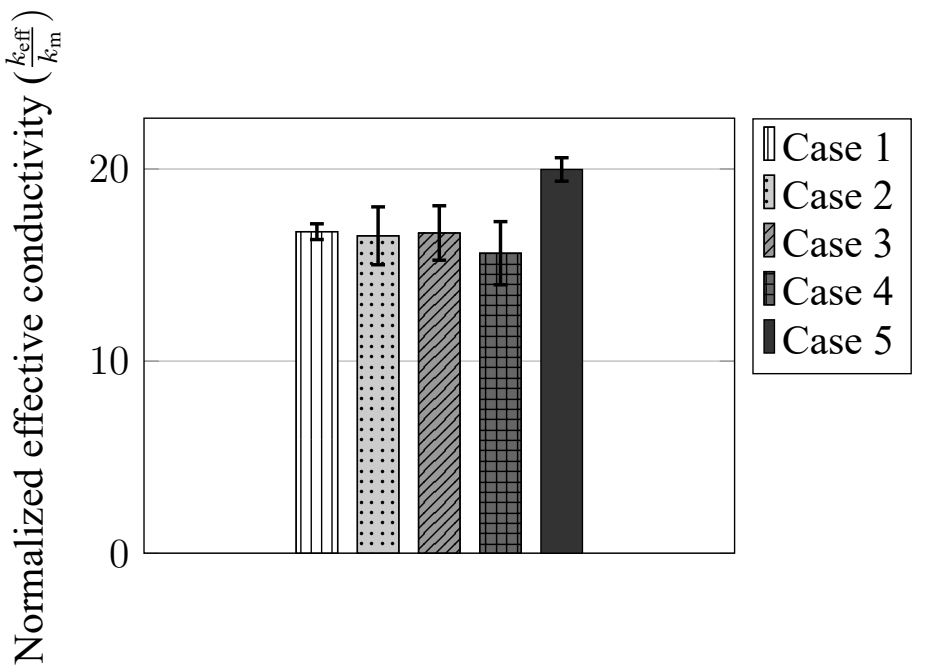

Fig. 5: Effective conductivity of the composite for four cases of randomly-oriented fiber distributions and an aligned fiber distribution
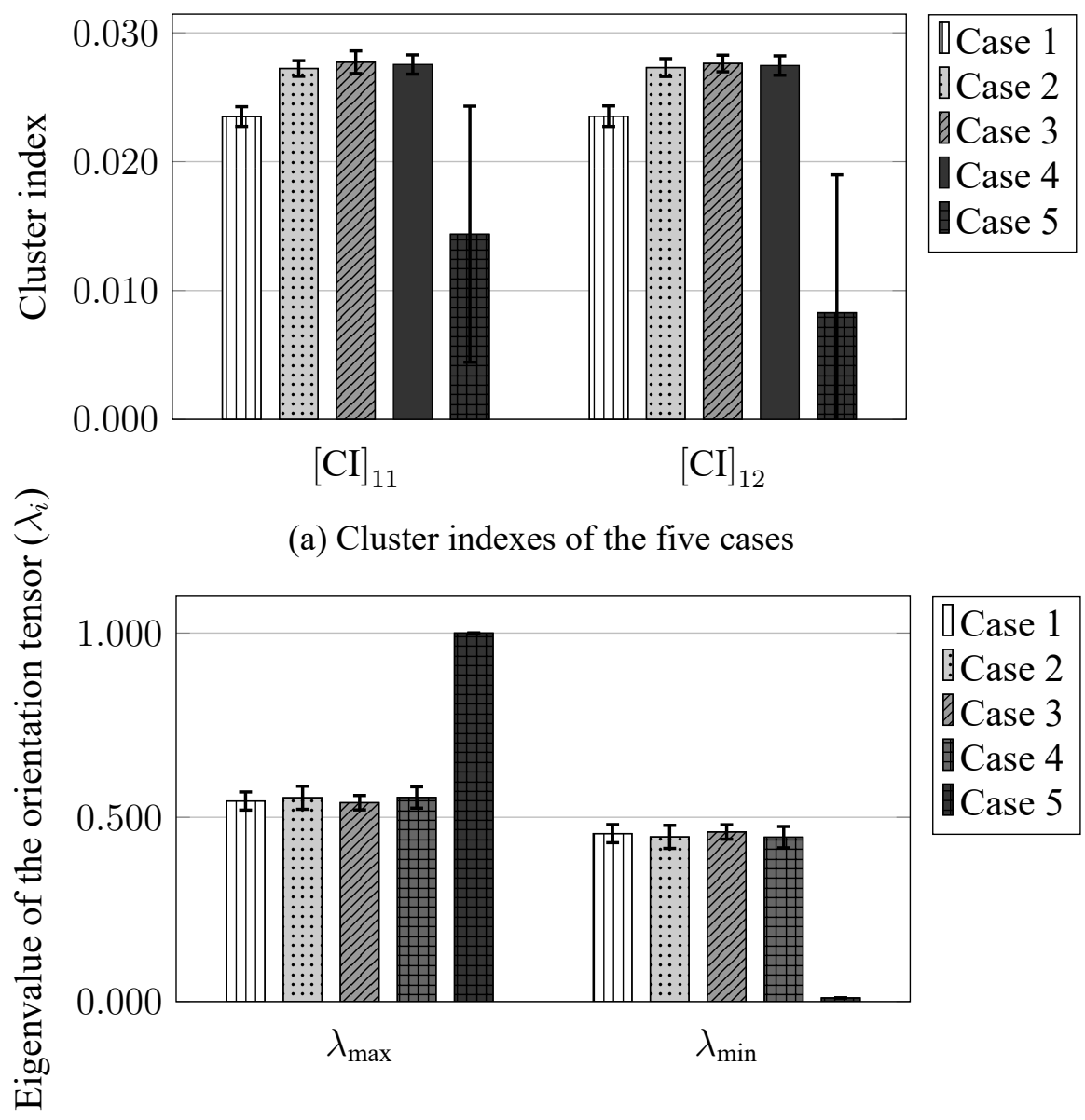

(b) Principal values of the five cases

Fig. 6: Summary of the results for parametric analyses 
Comparing the cluster indexes does not show a significant difference between all cases except the aligned fiber case which has the lowest clustering value. This denotes the alignment of the fibers which also results in close-to-zero clustering values with a rather large deviation, see Fig. 6a. In addition, all the partitioned cases show higher clustering than randomly distributed fibers without any partitions (case 1). This is another consequence of preventing cross-partition fiber extensions.

Ellipses were used to illustrate the results of principal component analyses. An skewed ellipse denotes a highly oriented state along the maximum principal direction which in the extreme case becomes a line along the same direction; this is what happened in the case of the aligned fibers. In contrast, the ellipse becomes a circle when there is no preferred orientation state. A couple of typical models are shown in Fig. 7 to illustrate this concept. In the conducted analyses, since all the random cases were uniformly distributed, values of the same order of magnitude were acquired for the eigenvalues, see $6 \mathrm{~b}$. However, the random nature of the distributions can be noted by the obtained diverse principal directions, see Table 2.

In conclusion, the finite element method along with the principal component analysis aided to reveal additional information about the anisotropic nature of the randomly-oriented short fibers. In the randomly-oriented cases, the effective conductivity values were mainly under the influence of the fiber volume fraction rather than any clustering effects. However, an extreme aligned case is distinguishable by considering the principal directions and their values. In addition, such eigenvalues can be used to distinguish a uniform fiber distribution from a clustered one. Fiber distribution through partitioning showed a resistance against conductivity in the case of non-uniform distribution of the fibers between partitions. This resulted in more local concentration of the fibers and slightly reduced RVE conductivity. Further investigating the effect of cross-partition penetration is recommended in the future studies. Finally, since no direct relation is discovered between the clustering index and the effective conductivity of the RVE, a more local investigation of each case is recommended to consider the effects of the internal anisotropic structure.

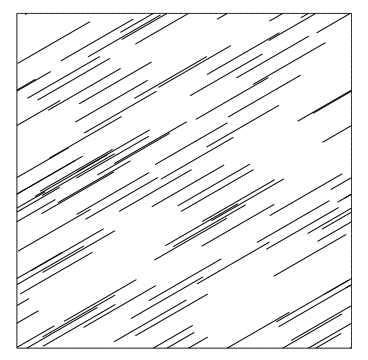

(a) A typical aligned fiber distribution

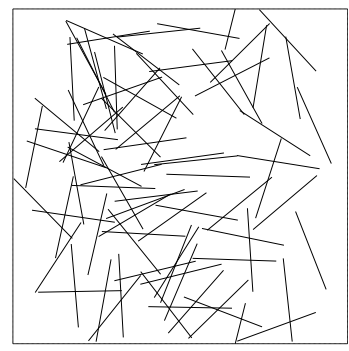

(c) A first case uniform random fiber distribution

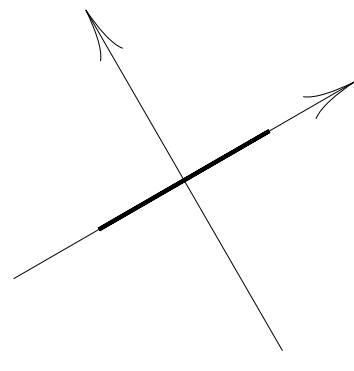

(b) Ellipse corresponding to the aligned distribution

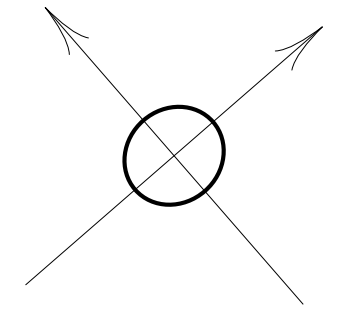

(d) Ellipse corresponding to the random distribution

Fig. 7: Denoting the principal directions and principal values by ellipses 


\section{References}

[1] H. Altenbach, J. Altenbach, and W. Kissing: Mechanics of composite structural elements (Springer, Berlin and Heidelberg 2010).

[2] R. M. Jones: Mechanics of composite materials (Taylor \& Francis, Philadelphia, Pa. and London, 1999).

[3] Z. Lu, Z. Yuan, and Q. Liu: Comp. Mater. Sci. Vol. 90 (2014), p. 123

[4] Z. Javanbakht, W. Hall, and A. Öchsner: Defect Diffus. Forum Vol. 372 (2016), p. 208

[5] Z. Javanbakht, W. Hall, and A. Öchsner: Defect Diffus. Forum Vol. 370 (2016), p. 177

[6] Y. Kataoka and M. Taya: JSME Int. J. A-Mech. M. Vol. 43.1 (2000), p. 46

[7] D. A. Jack and D. E. Smith: J. Rheol. Vol. 49.5 (2005), p. 1091

[8] T. C. Tszeng: Compos. Part B-ENG. Vol. 29.3 (1998), p. 299

[9] A. Tewari et al.: Acta Mater. Vol. 52 (2004) p. 307

[10] F. Folgar and C. L. Tucker: J. Reinf. Plast. Comp. Vol. 3 (1984) p. 98

[11] K. Kanatani: Int. J. Eng. Sci. Vol. 22 (1984) p. 149

[12] S. G. Advani and C. L. Tucker: J. Rheol. Vol. 31 (1987) p. 751

[13] S. G. Advani and C. L. Tucker: J. Rheol. Vol. 34 (1990) p. 367

[14] K. S. Lee et al.: J. App. Polym. Sci. Vol. 88 (2003) p. 500

[15] V. Müller et al.: J. Compos. Mater. Vol. 50 (2015) p. 297

[16] Z. Fan and S. G. Advani: Polymer Vol. 46 (2005) p. 5232

[17] C. Yang, H.-X. Huang, and K. Li: Polym. Composites Vol. 31 (2010) p. 1899

[18] S. Ranganathan and S. G. Advani: J Polym. Sci. Pol. Phys. Vol. 28 (1990) p. 2651

[19] A. Bertram and R. Glüge: Solid Mechanics (Springer International Publishing, Cham 2015)

[20] A. Öchsner and M. Merkel: One-dimensional finite elements: an introduction to the FE method (Springer, Berlin and Heidelberg 2013)

[21] A. Öchsner: Computational statics and dynamics: an introduction based on the finite element method (Springer, Singapore 2016)

[22] Z. Javanbakht and A. Öchsner: Computational statics revision course (Springer, Cham 2018)

[23] Z. Javanbakht and A. Öchsner: Advanced finite element simulation with MSC Marc: Application of user subroutines (Springer, Cham 2017)

[24] S. Kalpakjian and S. R. Schmid: Manufacturing engineering and technology (Prentice Hall, New York and Toronto 2010)

[25] Z. Javanbakht, W. Hall, and A. Öchsner: Materialwiss. Werkst. Vol. 48 (2017) p. 456 
[26] M. R. Islam and A. Pramila: J. Compos. Mater. Vol. 33 (1999) p. 1699

[27] T. Fiedler et al.: Computational Materials Science, Vol. 47 (2009) p. 314

[28] Z. Javanbakht, W. Hall, and A. Öchsner, in: Improved Performance of Materials, edited by A. Öchsner, and H. Altenbach, volume 72 of Advanced Structured Materials, chapter, 16, Springer (2018). 